\title{
Caminabilidad: El derecho a una ciudad sustentable e inclusiva
}

\author{
Walkability: The sustainable and inclusive city right
}

\author{
Nora Nacif ${ }^{1}$, Lorena Nieto ${ }^{1}$ \\ noranacif@faud.unsj.edu.ar; nietolorena@yahoo.com.ar \\ ${ }^{1}$ Gabinete de Investigaciones Urbanas (GIUR), del Instituto Regional de Planeamiento y Hábitat (IRPHA). \\ Facultad de Arquitectura, Urbanismo y Diseño (FAUD). Universidad Nacional de San Juan (UNSJ)
}

Enviado 30/03/2021 - Aceptado 14/06/2021

Nacif, Nora; Nieto Lorena (2021). "Caminabilidad: El derecho a una ciudad sustentable e inclusiva". En Proyección: estudios geográficos y de ordenamiento territorial. Vol. XV, (29). ISSN 1852 -0006, (pp. 104 - 129). Instituto CIFOT, Universidad Nacional de Cuyo. Mendoza. 


\section{Resumen}

Este trabajo se enmarca en un proyecto de investigación desarrollado por investigadores multidisciplinares, dentro de una línea de investigación sobre el desarrollo urbano sustentable. Entendiendo por tal, al que considera como ejes del desarrollo a la equidad social, la eficiencia económica y la preservación del ambiente urbano.

Dentro de este marco, el presente trabajo propone un acercamiento al estudio de la caminabilidad como una herramienta para entender la ciudad desde la mirada de la inclusión social, en el contexto general del concepto de Derecho a la Ciudad y con anclaje empírico en la ciudad de San Juan, Argentina.

Caminabilidad es el término empleado para evaluar qué tan amigable es un área urbana para desplazarse a pie para todos los transeúntes, pero sobre todo para aquellos que tienen dificultades para movilizarse por diversos motivos relativos a su condición física, género o edad.

Metodológicamente el trabajo se ordena en tres etapas operativas. La primera, consiste en la revisión y análisis de antecedentes relacionados al tema, en la segunda se plantea un relevamiento de la situación del área de estudio, y por último, se proponen indicadores para valorar las condiciones de caminabilidad de algunas áreas de la ciudad de San Juan, seleccionadas al efecto.

Palabras clave: Urbanismo Sustentable - Inclusión Social- Indicadores

\section{Abstract}

This work is part of a research project developed by multidisciplinary researchers, within a line of research about sustainable urban development. That concept considers social equity, economic efficiency and the preservation of the urban environment as axes of development.

The present article proposes an approach to the study of walkability as a way of understanding the city from a social inclusion perspective in the general context of the Right to the City concept. The empirical anchoring is made in San Juan city, Argentina.

Walkability is the word used to evaluate how friendly an urban area is to walk for all pedestrians. Especially for those who have difficulty getting around due to various reasons related to their physical condition, gender or age.

Methodologically the work is ordered into three operational stages: The first part is the review and analysis of antecedents related to the subject. The second part is focused on a survey of the study area situation, and finally, indicators are proposed to assess the walkability conditions of some areas selected for this purpose in San Juan city.

Key words: Sustainable Urbanism - Social inclusion- Indicators 


\section{Introducción}

Si bien Lefebvre (1968) planteó el concepto de Derecho a la Ciudad para debatir (u objetar) el sistema capitalista y sus implicancias en la ciudad, "hoy forma parte del discurso de un conjunto bastante amplio de organismos internacionales, académicos, tecnócratas y movimientos sociales urbanos bajo distintos significados y matices" (Carrión F.y Dammert-Guardia M., 2019).

El concepto Derecho a la ciudad, interpela inmediatamente respecto a qué tipo de ciudad se hace referencia, o, aún más, a que aspectos de la ciudad. Podría referirse al derecho a la ciudad latinoamericana en general, aunque es posible que esta generalización conlleve al uso de conceptualizaciones confusas y banales, derivadas del exceso de menciones que ha tenido y a las enormes diferencias regionales que se verifican en toda la extensión latinoamericana.

Para Lefebvre (1969; 1972), "el problema urbano es un problema revolucionario; y la revolución urbana, en sus términos, constituye una parte de la revolución total" (Nuñez, A. 2016). En ese complejo entretejido de conceptos con fuerte contenido político y posicionamiento epistemológico, podría resultar inadecuado reducirlo y/o recortarlo, descontextualizándolo de su integralidad. Aun así, y haciéndose cargo de ese riesgo, sigue resultando de interés el alto potencial que tiene el concepto como generador de interpretaciones críticas sobre la ciudad y como base para elaboraciones teóricas y empíricas de diversos estudios urbanos, apelando a atributos propios de la ciudad, tales como, la accesibilidad, los espacios públicos, los equipamientos e infraestructuras. Es decir aspectos básicos de la cuestión urbana, pueden ser abordados a modo de derechos de la población, como factores para la integración social y la cohesión social urbana, generando condiciones para la construcción de ciudadanía.

En este sentido, el derecho a la ciudad se convierte en un imperativo colectivo, que trasciende la esfera individual en cuanto a las posibilidades de acceso a los recursos que una ciudad ofrece. Más bien, sugiere e interpela sobre las transformaciones urbanas requeridas para minimizar o, en el mejor de los casos, eliminar elementos o aspectos matrices de inequidad y disgregación entre los diversos sectores urbanos. "La noción del derecho a la ciudad hace referencia a un derecho colectivo que se integra por todos los derechos que una ciudad garantiza a quienes en ella habitan, trabajan y transitan" (Ziccardi, A. 2017). 
En este contexto, es oportuno citar la Carta Mundial por el Derecho a la Ciudad (Coalición Internacional para el Derecho a la Ciudad, 2004) que busca recoger los compromisos y medidas que deben ser asumidos por la sociedad civil, los gobiernos locales y nacionales, parlamentarios y organismos internacionales para que todas las personas vivan con dignidad en las ciudades. En este documento sobre el Derecho a la Ciudad, se amplía el tradicional enfoque sobre la mejora de la calidad de vida de las personas centrado en la vivienda y el barrio hasta abarcar la calidad de vida a escala de ciudad y su entorno rural, como un mecanismo de protección de la población que vive en ciudades o regiones en acelerado proceso de urbanización. También establece entre otros asuntos, que la movilidad (como concepto multiescalar e integrador de diferentes modos de transitar) constituye un elemento central para ejercer dicho derecho, tal como consigna en el Art. XIII, punto 3, donde refiere que las ciudades deben promover la remoción de barreras arquitectónicas, la implantación de los equipamientos necesarios en el sistema de movilidad y circulación y la adaptación de todas las edificaciones públicas o de uso público y los locales de trabajo y esparcimiento para garantizar la accesibilidad de las personas con discapacidad. Es pertinente mencionar además, el Programa Hábitat de la Organización de Naciones Unidas -ONU-, que recupera los postulados anteriores y promueve el desarrollo de asentamientos humanos sostenibles desde el punto de vista social y medioambiental, basándose en los Objetivos del Desarrollo Sostenible (ODS), adoptada en Hábitat III para la Agenda 2030 (ONU-Hábitat, 2016).

Converge en estas consideraciones el derecho a una ciudad sustentable:

Pensar en términos de sustentabilidad, permite entender el desarrollo urbano desde una perspectiva abarcadora y "relacional" de los distintos aspectos que ello involucra. Esto es, la equidad social, la eficiencia económica y la preservación del ambiente. La ciudad aparece entonces como el escenario que permite y coadyuva a la interrelación de los subsistemas Natural y Antropizado. (Nacif N, Espinosa M., Martinet M., 2012)

En clave de derechos, una ciudad sustentable demanda una serie de condiciones para un ejercicio pleno e integral del mismo como el acceso al transporte, los servicios básicos, las nuevas tecnologías de información y comunicación, la seguridad ciudadana, la vivienda adecuada, el trabajo, entre otros (Ziccardi, A. 2017)

En esta línea, la mayoría de los autores pone el énfasis en el derecho al espacio público como lugar de convivencia, de interacción y cohesión social, donde debe prevalecer el respeto a las diversidades: culturales, de género, de condición física, entre otras. Por ello, en el derecho a una ciudad sustentable subyace el derecho al uso del espacio público como escenario que permite y coadyuva a la equidad social, uno 
de los pilares fundamentales del desarrollo urbano sustentable. El espacio público es el elemento estructural de un modelo de ciudad más sostenible. Es el espacio de convivencia ciudadana y forma, conjuntamente con la red de equipamientos y espacios verdes y de estancia, los ejes principales de la vida social y de relación (Agencia de Ecología Urbana de Barcelona, 2011).

Sin embargo, se puede afirmar que aunque la ciudad y todos sus atributos deben brindar igualdad de posibilidades de acceso a quienes la habitan, frecuentemente esto queda en el plano de las buenas intenciones y ello no se verifica en las oportunidades y en la satisfacción de necesidades de todos los grupos sociales.

"En una sociedad dada, la calidad de vida no es la misma para todos: distintos grupos sociales, sea por su nivel de renta, edad, sexo, raza, o lugar de residencia, tienen distintas necesidades y distintas posibilidades de acceso a los recursos y a los bienes sociales, ambientales y económicos que posibilitan el bienestar personal" (Sánchez de Madariaga I., 2008)

En este marco, el presente trabajo muestra parte de los resultados obtenidos en "Indicadores de Sustentabilidad Urbana: Estudio para un desarrollo urbano inteligente, sustentable e inclusivo", un proyecto de investigación realizado en el Gabinete de Investigaciones Urbanas dentro de una línea investigativa sobre el desarrollo urbano sustentable, financiado por la Secretaría de Ciencia y Técnica de la Universidad Nacional de San Juan, y con evaluación externa (2018/2019).

En ese proyecto se construyó un sistema de indicadores urbanos de sustentabilidad que constituyen el marco donde se desarrolla el trabajo que se presenta en este artículo. De modo que, aquí se pretende presentar un estudio sobre caminabilidad aplicado en algunos sectores de la ciudad de San Juan, Argentina, como modo de contribuir al derecho urbano a una mejor calidad de vida como aporte desde la mirada del desarrollo urbano sustentable. Entendiendo al derecho a la ciudad como el derecho al acceso a los más diversos bienes y servicios de carácter público propios de los ámbitos urbanos, lo que implica la consolidación de todos aquellos derechos necesarios para generar procesos de inclusión social.

Para ello se ha ordenado el trabajo en tres instancias metodológicas, que incluyen:

- Búsqueda y exploración de antecedentes: Revisión y recopilación de diversas visiones sobre el tema, que aportan autores e instituciones dedicadas al estudio de la temática.

- Descripción valorativa y selección del área de estudio. 
- Propuesta de indicadores de Caminabilidad Urbana en base a las etapas anteriores, que constituyan e interpreten las condiciones de sustentabilidad del área de estudio.

Comprometidos y convencidos de que "es necesario trabajar por ciudades inclusivas, que son aquellas que comprometen acciones y políticas públicas que garantizan la justicia social y la equidad para lograr ciudades habitables y sostenibles" (UN-Hábitat, 2016), enfatizamos a partir de lo expuesto que el "derecho a la ciudad" implica la construcción de "ciudades integradas" y que la caminabilidad debe enmarcase en esa mirada.

\section{Hacia una ciudad caminable}

Antecedentes del tema

En este trabajo la etapa de revisión y recopilación de antecedentes sobre caminabilidad, como en todo trabajo de investigación, se constituye en la base de posteriores instancias y se desarrolla en forma complementaria con la generación y organización de la información.

La caminabilidad de una comunidad puede definirse como un constructo multidimensional compuesto de diferentes factores que en conjunto constituyen un concepto teórico simple (Álvarez de Celis, Álvarez Insúa; Eguía; Pérsico; Belacin; Trípoli, 2014).

Esta multidimensionalidad conceptual genera la posibilidad de abordaje desde diversos enfoques, centrándose en algunos aspectos que determinan la potencialidad de un espacio para ser valorado como caminable.

Uno de ellos es el de la salud y los importantes beneficios que conlleva este hábito para la prevención y tratamiento de enfermedades. Otra mirada es la de la economía urbana, con la generación de actividades en torno a los lugares "caminables", con mayores rentas, menores costos de transporte y mayor estímulo a las actividades comerciales. No menos importante es el hecho de que caminar contribuye a disminuir la congestión vehicular y a la reducción de consumo de combustibles fósiles y las emisiones de gases de efecto invernadero, y así se lo aborda en otros trabajos (Gutiérrez López, Caballero Pérez, Escamilla Triana, 2019).

Es decir, que la caminabilidad conlleva conceptualmente valores intrínsecos a la urbanidad además de aportar beneficios de diversa índole y tenor: "El hecho de caminar y la ciudad van de la mano. Caminar ofrece una gama de beneficios que 
incluyen la movilidad, el ahorro de costos de consumo, la salud, la interacción social e importantes beneficios sociales" (Litman, 2007).

Durante gran parte del Siglo XX, la forma de movilidad basada en el automóvil recibió la mayor atención desde distintas esferas ligadas a su promoción y desarrollo, pero la creciente preocupación por sus consecuencias ambientales vinculadas al calentamiento global, los impactos sanitarios, sociales y económicos de estos sistemas de transporte, han renovado en los últimos 20 años el interés por la caminabilidad incentivando su estudio e investigación.

Como se explicó anteriormente, la multidimensionalidad del concepto ha generado estudios de diversa índole que abarcan multiplicidad de variables vinculadas a los objetivos de las disciplinas donde se generan. Por ello, y siguiendo las pautas metodológicas, a continuación, se presenta una tabla síntesis de la revisión de antecedentes sobre el tema (Tabla N¹). Se han seleccionado sólo algunos autores e instituciones que con sus estudios y aportes se consideran relevantes para este estudio de caminabilidad, por la profusa cantidad y calidad de la información revisada.

Tabla $\mathbf{N}^{\circ} 1$ - Antecedentes relevantes para el estudio de caminabilidad

\begin{tabular}{|c|c|c|}
\hline $\begin{array}{l}\text { Autor/Institución/Lugar- } \\
\text { Ámbito/Año }\end{array}$ & Título & Aportes del Estudio \\
\hline $\begin{array}{l}\text { Red CIMES-Ciudades Intermedias- } \\
\text { Cátedra UNESCO- } 2002\end{array}$ & $\begin{array}{l}\text { «Miradas a otros espacios urbanos: } \\
\text { las ciudades intermedias» }\end{array}$ & $\begin{array}{l}\text { Parámetros y dimensiones de análisis } \\
\text { para el estudio comparado de } \\
\text { ciudades de escala intermedia. }\end{array}$ \\
\hline $\begin{array}{l}\text { Agencia Ecología Urbana Barcelona- } \\
2011\end{array}$ & $\begin{array}{l}\text { «Sistema de indicadores y } \\
\text { condicionantes para } \\
\text { ciudades grandes y medianas» }\end{array}$ & $\begin{array}{l}\text { Indicadores clasificados según } \\
\text { ámbitos y fase de aplicabilidad. }\end{array}$ \\
\hline $\begin{array}{l}\text { Ministerio de Desarrollo Urbano- } \\
\text { Secretaría de Planeamiento, Buenos } \\
\text { Aires Ciudad- } 2011\end{array}$ & $\begin{array}{l}\text { "Índice sintético } \\
\text { de caminabilidad-Metodología» }\end{array}$ & $\begin{array}{l}\text { Aspectos conceptuales y } \\
\text { metodológicos para la construcción } \\
\text { del índice de caminabilidad en CABA. }\end{array}$ \\
\hline $\begin{array}{l}\text { Llop Torné, Josep María- Barcelona- } \\
2013\end{array}$ & $\begin{array}{l}\text { «Medir la sostenibilidad urbana a } \\
\text { escalas intermedias: Compacidad- } \\
\text { proximidad y habitabilidad- } \\
\text { accesibilidad» }\end{array}$ & $\begin{array}{l}\text { Fundamentos sobre la operatividad } \\
\text { de indicadores y parámetros de } \\
\text { medición para comprensión de la } \\
\text { escala intermedia. }\end{array}$ \\
\hline UN-Hábitat - 2015 & $\begin{array}{l}\text { "Transformar nuestro mundo: la } \\
\text { Agenda } 2030 \text { para el Desarrollo } \\
\text { Sostenible» }\end{array}$ & $\begin{array}{l}\text { Objetivos del Desarrollo Sostenible. } \\
\text { ODS. N } 11 \text { : Ciudades inclusivas, } \\
\text { seguras, resilientes y sostenibles. }\end{array}$ \\
\hline $\begin{array}{l}\text { Agampatian, Razmik- Nueva York- } \\
2014\end{array}$ & $\begin{array}{l}\text { "Uso de SIG para medir la } \\
\text { caminabilidad: un estudio de caso en } \\
\text { la ciudad de Nueva York» }\end{array}$ & $\begin{array}{l}\text { Aspectos conceptuales y } \\
\text { metodológicos para construcción del } \\
\text { índice de caminabilidad en NY. }\end{array}$ \\
\hline PNUD (ONU)- Argentina- 2017 & $\begin{array}{l}\text { «Información para el desarrollo } \\
\text { sostenible: Argentina y la Agenda } \\
2030 »\end{array}$ & $\begin{array}{l}\text { Panorama general del desarrollo } \\
\text { sostenible en Argentina. Índice para } \\
\text { caracterizar la situación a nivel } \\
\text { provincial. }\end{array}$ \\
\hline $\begin{array}{l}\text { Gutiérrez López, Caballero Pérez, } \\
\text { Escamilla Triana-Colombia- } 2019\end{array}$ & $\begin{array}{l}\text { "Índice de caminabilidad para la } \\
\text { ciudad de Bogotá» }\end{array}$ & $\begin{array}{l}\text { Aspectos conceptuales y } \\
\text { metodológicos para construcción del } \\
\text { índice de caminabilidad en Bogotá. }\end{array}$ \\
\hline
\end{tabular}

Fuente: Elaboración propia- 2020

Vol. XV - Año 2021 - pp.104 - 129 - Dossier - Proyección 
Breve reseña descriptiva de la ciudad de San Juan

El tema propuesto se plantea tomando como referente el caso del área metropolitana denominada Gran San Juan, localizada en el Valle de Tulum, Ullum y Zonda en la provincia de San Juan, Argentina. El Gran San Juan es una ciudad de escala intermedia y está conformado por las áreas urbanas de los departamentos Capital, Santa Lucía, Chimbas, Rivadavia, Rawson y sector norte de Pocito. (Figura 1).

\section{Figura №1- Gran San Juan}

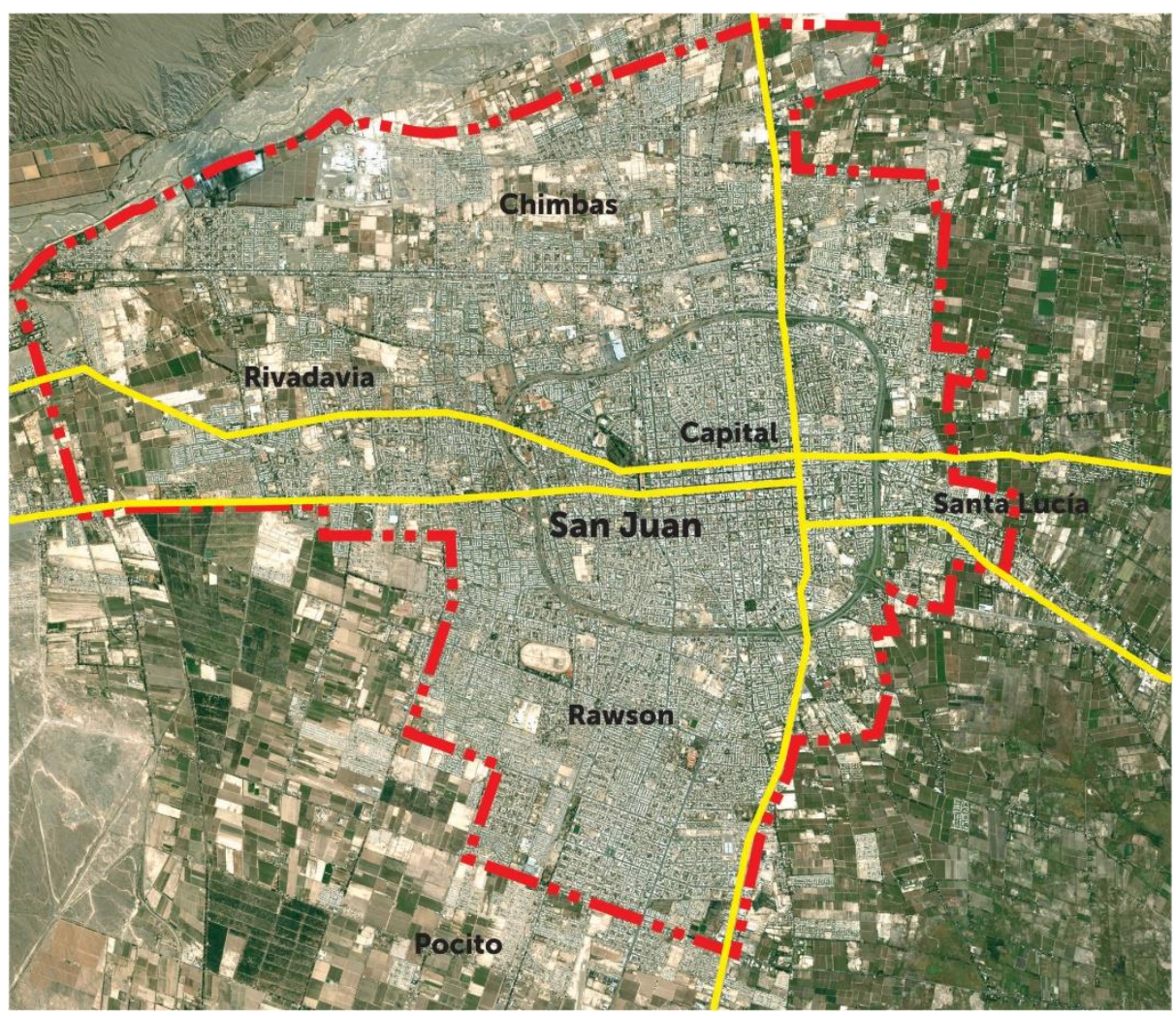

Fuente: Elaboración propia, en base a plano del geo portal UNIDE¹- 2021

En esta ciudad hay al menos dos aspectos a considerar en cualquier estudio urbano que se realice y que están referidos a dos condicionantes ineludibles de su medio natural: la aridez y la sismicidad. Es por ello que, desde la perspectiva del ambiente natural, se la puede caracterizar como una "ciudad oasis de zona sísmica". Esta particular situación lleva a considerar la necesidad de preservar su característica de "ciudad intermedia" la que es inescindible de la condición de "ciudad sustentable" desde la cual abordamos este trabajo (Nacif N, Espinosa M.P., Martinet M. 2012).

La ciudad fue reconstruida luego del terremoto de 1944 con estrictas normas de sismorresistencia de modo que "la crisis que produjo el terremoto originó un cambio

${ }_{1}^{1}$ UNIDE Unidad de Infraestructura de Datos Espaciales: https://geoportal.sanjuan.gob.ar/geoportal/\#10/-31.4820/68.5773 
morfológico y consecuentemente de imagen de la ciudad" (Sentagne, Solera, Roses y Laciar, 2010).

En el área central de la ciudad puede identificarse la mayor concentración de recursos a nivel edilicio, poblacional y de actividades económicas. Una serie de elementos urbanos constituidos por los principales espacios verdes y una red vial relativamente jerarquizada, configuran la estructura de una típica ciudad en cuadricula en el casco urbano que, en sus expansiones por fuera de él, toma diversas morfologías y tramas. (Roitman D, 1996). La diferencia no sólo es formal sino fundamentalmente funcional, por la alta dependencia que estos sectores adyacentes presentan respecto del área central.

\section{Selección e identificación del área de estudio}

El criterio seguido para la selección de los sectores urbanos considerados como anclaje empírico de este trabajo, fue por una parte, el de optar por aquellos que contaban con mayor cantidad de espacios de uso público y transitabilidad dentro del casco urbano, y en contraposición, otro sector de un barrio residencial localizado fuera del área central.

El clima, el paisaje y la cultura sanjuanina indican y refuerzan la idea de apropiación y vivencia de los espacios abiertos o accesibles en el más amplio sentido conceptual de espacio público. "El concepto de espacios abiertos para la apropiación social generalizada, históricamente ha demostrado ser la esencia de esta ciudad...la nueva ciudad remodelada a partir del terremoto respetó esta "esencia" y enriqueció la trama de los espacios públicos". (Roitman D. 1996).

En consecuencia, se definen dos áreas como unidades espaciales en términos de estructura e identidad urbano-territorial y que abarcan distintas áreas urbanas caminables:

1) Área Central, comprende: microcentro con predominancia del uso comercial, eje cívico- institucional, espacios verdes principales.

2) Área de Cercanía, comprende: zonas adyacentes al área central, con predominio de uso residencial, mixturado con comercio diario o periódico barrial y espacios verdes municipales o barriales.

Asumiendo esos criterios y en ese marco de referencia, se seleccionan los sectores para el estudio y aplicación del indicador de caminabilidad, ajustándose al esquema metodológico planteado (Figura $\mathrm{N}^{\circ} 2$ ): 
1- En el Área central, se determina:

1.1- Microcentro de San Juan, comprende: área peatonal comercial; eje cívicoinstitucional (Av. Central Ignacio de la Roza), Plaza Central 25 de Mayo.

2- En el Área de cercanía:

2.1- Barrio del Carmen

Figura N²: Ubicación áreas de estudio

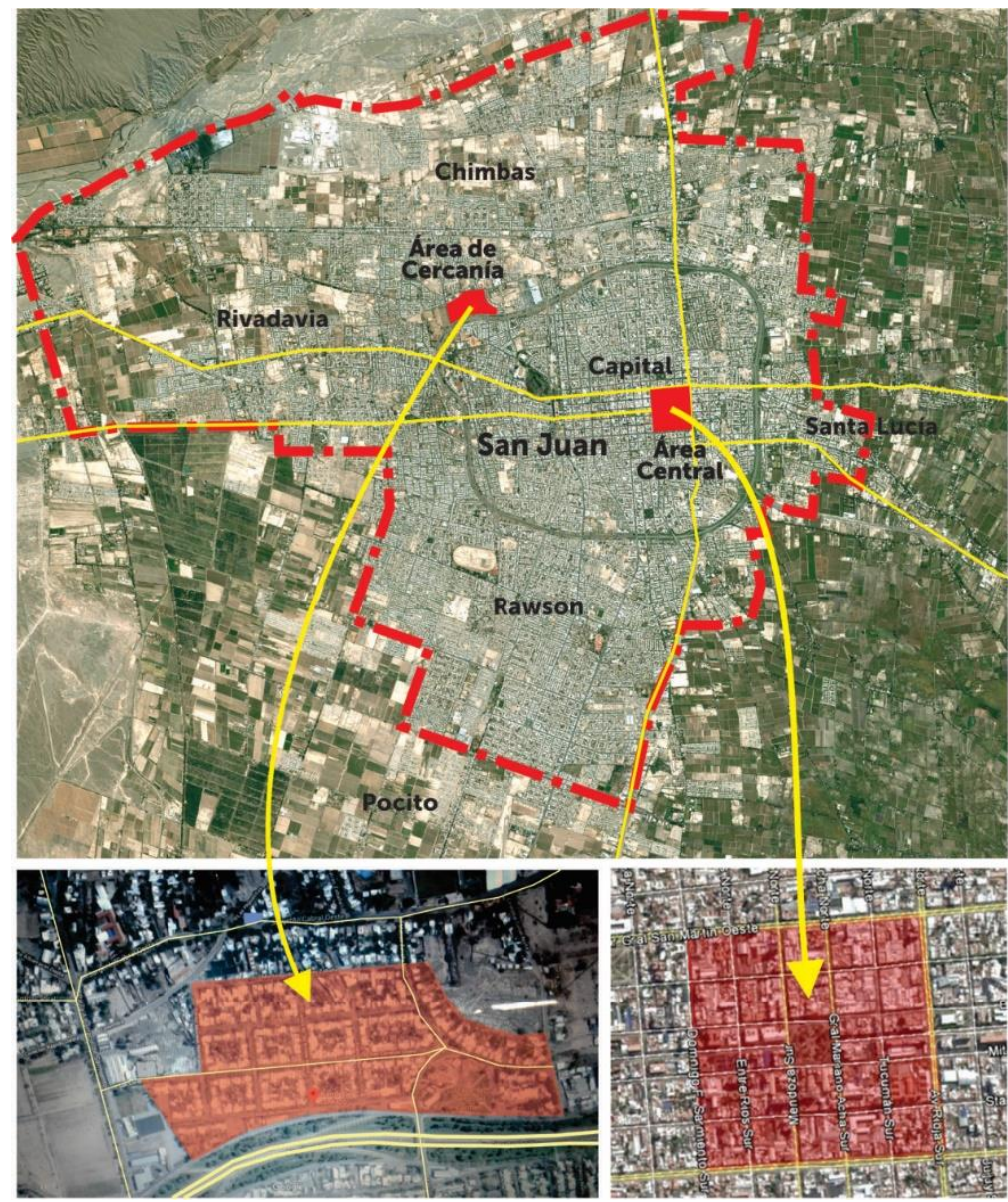

Fuente: Elaboración propia, en base a plano Google y del geo portal UNIDE- 2021

\section{Propuesta de Indicador de Caminabilidad}

La caminabilidad como derecho a la ciudad y su estudio como aporte al desarrollo sustentable, es el principio y marco conceptual desde el que se propone la construcción de un indicador para su "operativización", que se inserta en un sistema integral de indicadores de sustentabilidad urbana.

En esta instancia resulta procedente destacar la importancia de la consideración de indicadores en el proceso integral de una planificación urbana que apunte a la sustentabilidad. "La aproximación a la problemática del desarrollo urbano sustentable 
requiere de diversas herramientas tales como los indicadores, que representan una forma de medir e identificar los conflictos de la ciudad, en forma concreta y espacializada" (Nacif N, Espinosa M., Martinet M. (2012)

En ese sentido, un estudio de indicadores urbanos posee una doble finalidad en la temporalidad de su utilización: la posibilidad de mejorar la situación actual y el desarrollo de la capacidad de respuesta para que la situación futura sea también mejor (Figura 2). Los indicadores se convierten así en herramientas de monitoreo permanente para la detección de conflictos y son un aporte para avanzar a escenarios deseables.

Figura N³- Utilización de Indicadores

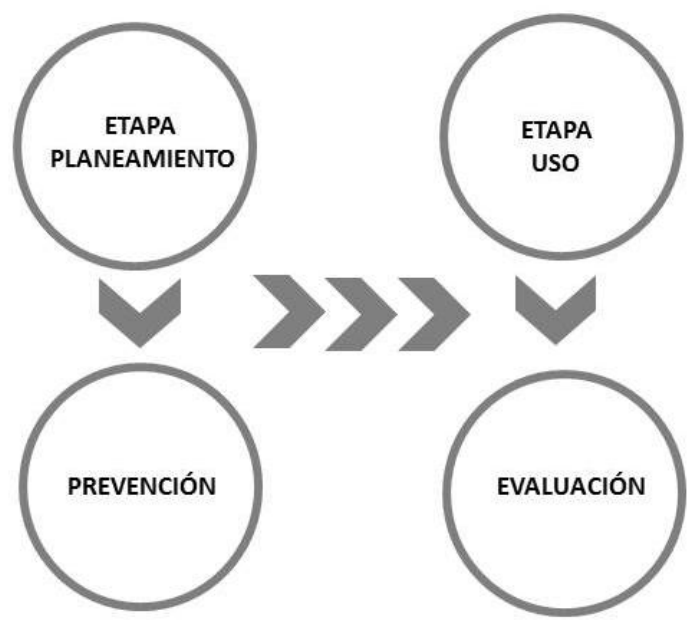

Fuente: Elaboración Propia- GIUR

El objetivo subyacente es la mejora continua en la calidad de vida de la población, atendiendo a la valoración que arrojan estos instrumentos. De modo que, los indicadores urbanos son elementos de información que intentan reflejar el estado real de la ciudad objeto de estudio. Facilitan el proceso de análisis y otorgan claridad para efectuar comparaciones. Por ello los indicadores adquieren gran valor en los procesos de evaluación y de toma de decisiones sobre los problemas urbanos" (Bär Sarda A., 2009).

"Un indicador urbano es un conjunto ordenado de variables sintéticas cuyo objetivo es proveer de una visión totalizadora respecto a los intereses relativos a la realidad urbana de que se trate" (Rueda Palenzuela, S. 1999).

La lógica de construcción de un indicador sería, a) Construcción abstracta del problema, para establecer las regularidades y plantearlo como un problema de 
medida, b) Tomar aspectos o dimensiones deducidas analíticamente o por sus interrelaciones empíricas y c) Definir los indicadores (Gutiérrez D. 2009).

La propuesta del indicador de caminabilidad que presentamos, se enmarca en el desarrollo de un sistema de indicadores urbanos de sustentabilidad, elaborados para la ciudad de San Juan, en el Gabinete de Investigaciones Urbanas, FAUD-UNSJ, tal como se explicitó anteriormente:

"Esta propuesta se realiza desde una perspectiva sistémica, que se centra en los aspectos físicoespaciales, socio-económicos y ambientales, que constituyen los principales subsistemas de acuerdo a los principios del desarrollo urbano sustentable. Cada subsistema se desagrega en ámbitos y sub-ámbitos que a su vez articulan y agrupan a los diversos indicadores principales e indicadores de interrelación". (Nacif, N. 2017)

Tabla $\mathbf{N}^{\circ}$ 2: Sistema de Indicadores de Sustentabilidad Urbana.

\begin{tabular}{|c|c|c|c|}
\hline SUBSISTEMA & ÁMBITO & SUB-ÁMBITO & INDICADOR \\
\hline \multirow{3}{*}{$\begin{array}{l}\text { FíSICO ESPACIAL } \\
\text { Y FUNCIONAL }\end{array}$} & Ocupación del suelo & Intensidad de Uso & $\begin{array}{l}\text {-Tamaño y forma de la ciudad } \\
\text {-Compacidad } \\
\text {-Densidad constructiva } \\
\text {-Densidad de población }\end{array}$ \\
\hline & \multirow[b]{2}{*}{$\begin{array}{l}\text { Espaciopúblicoy } \\
\text { habitabilidad }\end{array}$} & $\begin{array}{l}\text { Bienes culturales } \\
\text { tangibles }\end{array}$ & $\begin{array}{l}\text {-Patrimonio urbano } \\
\text {-Patrimonio arquitectónico }\end{array}$ \\
\hline & & Viario urbano & $\begin{array}{l}\text {-Accesibilidad } \\
\text {-Mobiliariourbano } \\
\text {-Confort acústico } \\
\text {-Confort térmico } \\
\text {-Contaminación visual }\end{array}$ \\
\hline \multirow{4}{*}{$\begin{array}{l}\text { SOCIO ECONÓMICO } \\
\text { CULTURAL }\end{array}$} & \multirow[t]{2}{*}{$\begin{array}{c}\text { Accesibilidade } \\
\text { Integración Social }\end{array}$} & Cohesión Social & $\begin{array}{l}\text {-Identidad de la ciudad } \\
\text {-Dotaciónequipamientos públicos } \\
\text {-Acceso a vivienda económica } \\
\text { - Inclusión sociale integración } \\
\text { personas con discapacidad } \\
\text { - Cominabilidad }\end{array}$ \\
\hline & & Educación & $\begin{array}{l}\text {-Nivel de instrucción de la } \\
\text { población } \\
\text {-Educación ambiental }\end{array}$ \\
\hline & Complejidad Urbana & Equilibrio de Actividades & $\begin{array}{l}\text {-Densidad de actividades terciarias } \\
\text {-Densidad de uso residencial } \\
\text {-Dotación de equipamiento urbano } \\
\text {-Transformación del paisaje urbano }\end{array}$ \\
\hline & Movilidady Servicios & $\begin{array}{l}\text { Desplazamiento de la } \\
\text { población }\end{array}$ & $\begin{array}{l}\text {-Proximidad a equipamientos } \\
\text {-Proximidad a transporte público } \\
\text {-Frecuenciadel transporte público } \\
\text {-Estacionamiento }\end{array}$ \\
\hline \multirow{3}{*}{ AMBIENTAL } & Riesgos Urbanos & $\begin{array}{l}\text { Vulnerabilidad ante } \\
\text { fenómenos ambientales }\end{array}$ & $\begin{array}{l}\text {-Vulnerabilidad Fisica } \\
\text {-Vulnerabilidad Funcional } \\
\text {-Vulnerabilidad Social }\end{array}$ \\
\hline & Verde Urbano & $\begin{array}{l}\text { Espacios verdesy } \\
\text { arbolado urbano }\end{array}$ & $\begin{array}{l}\text { Superficie verde porhab } \\
\text {-Distancia a espacio verde } \\
\text {-Cantidad de espacios verdes } \\
\text { - Cubiertas verdes }\end{array}$ \\
\hline & Metabolismo urbano & $\begin{array}{l}\text { Generación y manejo de } \\
\text { energía, agua y residuos }\end{array}$ & $\begin{array}{l}\text {-Consumo energia tradicionaly } \\
\text { renovable } \\
\text { - Consumo hídrico } \\
\text {-Gestión de residuos }\end{array}$ \\
\hline
\end{tabular}

Fuente: Elaboración propia. Gabinete Investigaciones Urbanas-GIUR-FAUD- 2015

El indicador de caminabilidad propuesto, corresponde en este sistema al subsistema Socio-económico-cultural, ámbito Accesibilidad e Integración social, sub-ámbito Cohesión Social. Sin embargo, se debe aclarar que este sistema contiene una 
diversidad de indicadores con connotaciones particulares y que su observación para obtener una mirada integral de la cuestión urbana, puede conducir a análisis que relacionen los tres subsistemas con sus ámbitos de atribución. De modo tal, que hay indicadores que trasponen la incumbencia de un solo subsistema y pueden ser expresión de dos o más de ellos, como es el caso del indicador de caminabilidad propuesto. "Esta interrelación permanente entre indicadores y subsistemas, indica una complejidad que no puede quedar limitada a la simple "suma" de los indicadores parciales sino que la riqueza de los resultados se advierte en una interpretación sistémica e integrada" (Nacif N, 2017).

Es oportuno aclarar que para el correcto funcionamiento de este sistema se trabaja con indicadores que, en próximas fases investigativas podrían constituir Índices, amalgamando temáticas comunes a los subsistemas, a sus ámbitos y/o sub-ámbitos. "Algunos sistemas de indicadores no se satisfacen con seleccionar una o más variables descriptivas de un fenómeno como mecanismo de síntesis de la información para tomar decisiones, sino que fusionan la información contenida en varias de ellas en una sola expresión numérica. La magnitud resultante de tal fusión se denomina índice" (Leva G., 2005).

\section{Objetivo del Indicador}

Evaluar las condiciones para caminar en áreas urbanas de manera confortable y segura, identificando situaciones críticas que sean susceptibles de ser intervenidas para lograr que el entorno urbano tenga las características óptimas para su recorrido a pie.

\section{Determinación de variables y sus parámetros}

En continuidad con el esquema metodológico, se determinan las variables y sus parámetros fundamentales para la estructuración y diseño del indicador. Por ello, para analizar el impacto de las variables de caminabilidad, es importante identificar aquellas que sean pertinentes, medibles, y fáciles de comprender (Gutiérrez Lopez et al, 2019).

En ese sentido, se considera que las personas son más propensas a caminar cuando tienen, dentro de un radio de aproximadamente $1 \mathrm{~km}$. respecto de sus viviendas, establecimientos educativos, edificios de oficinas, locales comerciales, restaurantes, parques, supermercados y edificios institucionales. Es decir que las condiciones del área a recorrer sean adecuadas, atractivas y/o con una manifiesta conectividad operativo-funcional: 
De acuerdo con diversos autores (J.Jacobs,1961; A. Jacobs, 1995; Farr, 2008), las razones que determinan la decisión de las personas a caminar están dadas por la variedad de usos y actividades durante el recorrido a realizar, la proximidad del lugar de residencia a dichos usos, la accesibilidad y posibilidad de realizar múltiples conexiones entre distintos destinos, la calidad estética del espacio, la existencia de condiciones que faciliten la experiencia del caminar (mobiliario, áreas de descanso, senderos peatonales, mantenimiento adecuado, vegetación) y la posibilidad de socializar con otras personas (En Álvarez de Celis, F, et. al. 2014).

Enmarcados en lo anteriormente citado, se establecieron tres variables básicas, con sus parámetros, que conjugan las principales características urbanas que permiten identificar un área como "caminable":
a) Mixtura de usos-
b) Confort peatonal-
c) Calidad ambiental

a) Mixtura de usos: Referida a la heterogeneidad en los usos del suelo identificables en un sector urbano y que coexisten sin generar conflictos en la dinámica urbana, favoreciendo las relaciones y actividades socio- económicas y culturales. Esta característica facilita el desarrollo de la vida cotidiana y fomenta el desplazamiento de las personas a pie (o en bicicleta). El grado de mixticidad ${ }^{2}$ de usos y funciones implantadas en un determinado territorio, evidencia la complejidad urbana que es el reflejo de las interacciones que se establecen en la ciudad (Agencia de Ecología Urbana de Barcelona, 2011). Eliminar las barreras de zonificación y adoptar usos múltiples compatibles puede generar además, beneficios sociales mejorando la accesibilidad a servicios y equipamientos (ONU-Hábitat, 2021).

Los parámetros fundamentales que permiten su análisis y valoración, están vinculados con la concentración de actividades, la diversidad de actividades, la densidad de población y la distancia o proximidad a equipamientos y/o destinos frecuentes.

b) Confort y seguridad peatonal: Refiere a las condiciones del entorno para garantizar un recorrido confortable y seguro. Esto es, las características que hacen que determinada área sea adecuada para desplazarse y recorrerla a pie, por su nivel de accesibilidad, infraestructura y el de seguridad. La llamada "vigilancia informal" que consiste en ver y ser visto (Bar Sarda A., 2009). La consideración de recorridos seguros, en cuanto a su iluminación y con el amparo de la presencia de otros transeúntes es fundamental para evitar la inseguridad y/o la sensación de riesgo que, si bien en la actualidad atraviesa a toda la sociedad, es más evidente en las mujeres, en los niños y en los adultos mayores.

\footnotetext{
${ }^{2}$ Mixticidad se emplea como un concepto que involucra un proceso complejo, generado no sólo por la existencia de diversos factores sociales y espaciales sino que además, se verifica interacción entre ellos.
} 
"La violencia en las ciudades, la que se vive y la que se percibe, constituye un tema emergente, complejo y preocupante, que ocupa las agendas de la sociedad civil y las de los gobiernos. Es de destacar, que estas violencias no son vividas ni sentidas de igual manera por toda la ciudadanía: no es igual ser mujer que hombre, ni joven que adulto mayor..." (Falú,A., 2014).

Esta característica está estrechamente vinculada al diseño urbano en cuanto a los criterios físico-espaciales, funcionales y además, estéticos. Hay una "necesidad de satisfacción estética" que juega un importante papel en el funcionamiento del individuo y que debe tenerse en cuenta en el diseño urbano porque tiene una gran importancia para la actuación e identificación del sujeto en la ciudad. (Corraliza Rodriguez, Berenguer, Martín, 2006).

Por ello los parámetros para su evaluación se basan en la dotación de mobiliario urbano, las dimensiones y el estado de las veredas, la existencia de señalética urbana, la iluminación, la existencia de protección frente al tránsito automotor, la continuidad y claridad de los recorridos y la conectividad de la red peatonal (Álvarez de Celis, F, et. al. 2014).

c) Calidad ambiental: Si bien esta variable posee una gran amplitud conceptual, parece oportuno acotarla a las condiciones que hacen que el ambiente urbano sea saludable, agradable y propicio para promover el hábito de caminar. El ruido y los contaminantes atmosféricos constituyen un riesgo de primer orden para la calidad ambiental y la salud pública de las personas (Agencia de Ecología Urbana de Barcelona, 2011). Mientras que, el arbolado urbano es el principal elemento vegetal en las ciudades como elemento estructural de la biodiversidad en el ecosistema urbano y constituye una barrera cierta para los problemas ambientales relacionados con la contaminación del aire y la contaminación acústica. Su presencia en las ciudades, tanto en la red vial como en parques y plazas, supone una mejora de la calidad del espacio público: "El arbolado urbano contribuye a las mejoras de las condiciones ambientales de los espacios urbanos mediante la reducción de la temperatura en época estival, la oxigenación del aire y la absorción de contaminantes atmosféricos" (Álvarez de Celis, F, et. al. 2014).

En una ciudad de clima semiárido, muy caluroso en época estival como el de San Juan, la consideración del verde urbano es imprescindible como factor que proporciona "calidad ambiental" (GIUR- FAUD-UNSJ, 2014-2015).

Por lo tanto, los parámetros fundamentales para la evaluación de la calidad ambiental, se basan en la cantidad y estado del arbolado público presente en el sistema vial y la 
proximidad y accesibilidad de la población a los espacios verdes. También se considera como parámetro la contaminación ambiental, referida a la contaminación acústica y atmosférica. Ambos parámetros están estrechamente vinculados al tránsito automotor, que afecta al espacio urbano por las emisiones de contaminantes y residuos químicos, vibraciones y altos niveles sonoros que degradan la calidad del aire y el ambiente acústico (Álvarez de Celis, F, et. al. 2014). Cabe aclarar que la intensidad de tránsito y su impacto ambiental también dependen de la cantidad de líneas de transporte público que recorren las áreas a valorar y ello debe ser tenido en cuenta en la aplicación del indicador a cada caso de estudio.

\section{Diseño del Indicador}

En el diseño y construcción de un indicador, intervienen diferentes dimensiones y variables que se integran e interactúan entre sí, sintetizando la complejidad de los diversos componentes (Rueda Palenzuela, 1999).

En ese marco, la construcción y diseño del indicador de caminabilidad se fundamenta en la combinación de las variables identificadas y sus parámetros, con ponderaciones en un rango entre 0 y 1 , tomando como referencia los valores más críticos existentes (0) y los ideales (1). Además, se consideran las especificaciones contenidas en la Tabla №2.

Tabla N`3- Indicador de Caminabilidad

\section{INDICADOR DE CAMINABILIDAD}

\begin{tabular}{|c|c|}
\hline Tipo & Con tratamiento de datos \\
\hline Fuente de datos & $\begin{array}{l}\text { DPDU (Dirección de Planeamiento y Desarrollo } \\
\text { Urbano), Catastro, Municipios y Relevamientos in situ }\end{array}$ \\
\hline Escala / Unidad de cálculo & Variable (Radio Censal- Cuadra- Manzana- Sector) \\
\hline Periodicidad & Variable \\
\hline Tendencia deseable & 1 \\
\hline Fórmula & $\begin{array}{l}\text { Variables y parámetros intervinientes / Unidad de } \\
\text { cálculo }\end{array}$ \\
\hline
\end{tabular}

Fuente: Elaboración propia- GIUR-FAUD 2021

La unidad de cálculo es la manzana, la cuadra o un sector, dependiendo de la zona analizada y de la disponibilidad de información. La fórmula del indicador de caminabilidad, queda entonces establecida del siguiente modo: 


\section{Fórmula Indicador de Caminabilidad}

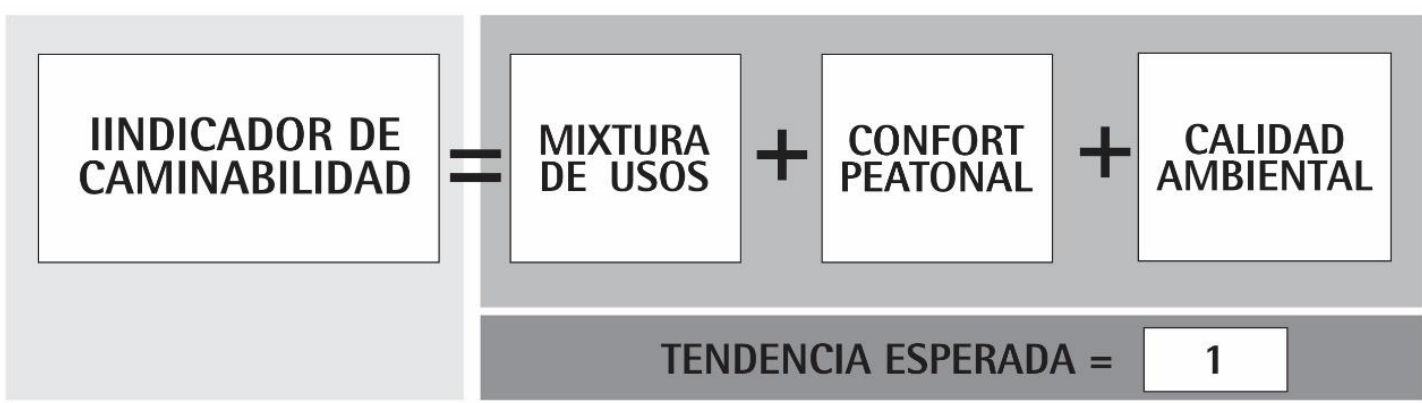

Fuente: Elaboración propia - GIUR -2020

A cada variable se le asigna un peso relativo dentro de la fórmula del indicador. "Los valores son referentes convencionales emanados de la reflexión, no del instinto" (Folch, R y Bru J- 2017). El criterio de asignación de esta ponderación refiere a la importancia de la variable y a la cantidad de parámetros necesarios para definirla. A su vez, cada variable se desagrega en los parámetros previamente determinados, a los que también se les asigna un peso dentro de cada variable.

La asignación de puntajes se realiza mediante el análisis de datos propios, por medio del uso de herramientas de sistemas de información geográfica (SIG), de datos obtenidos de la Municipalidad de la Capital de San Juan y del portal UNIDE (portal de información georrefenciada digital del Gobierno de la Provincia de San Juan)³.

Cabe aclarar que estos parámetros están asociados además con las fachadas y sus tipologías (permeabilidad, retiros, etc), los horarios de uso, la estación del año, la población flotante en determinadas zonas centrales y ello debe considerarse en la ponderación y aplicación del indicador en cada zona, de acuerdo a los objetivos del estudio a realizar.

Por otra parte, en este trabajo se asume la importancia de enmarcar este indicador en un estudio del sistema de movilidad general y de itinerarios o recorridos posibles, que pueden valorarse a través de diversas variables y parámetros asociados. Por ello, para la obtención de una mirada integral de la temática de la movilidad urbana, su consideración queda anclada al sistema general de indicadores y a las interrelaciones que puedan devenir en ese sistema, tal como se explicitó anteriormente.

En la Tabla $\mathrm{N}^{\circ} 3$ se observan los pesos establecidos para cada variable y sus parámetros en el Indicador total de Caminabilidad:

${ }^{3}$ UNIDE- https://geoportal.sanjuan.gob.ar/geoportal/\#7/-30.916/-68.621 
Tabla N`4- Indicador de Caminabilidad: Ponderación de Variables y Parámetros

\begin{tabular}{|c|c|c|}
\hline \multirow{5}{*}{ 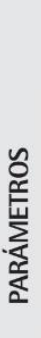 } & INDICADOR DE CAMINABILIDAD & Ponderación \\
\hline & VARIABLE: MIXTURA DE USOS & 0.3 \\
\hline & - Concentración de actividades & 0.1 \\
\hline & - Densidad de población & 0.1 \\
\hline & - Proximidad de equipamiento & 0.1 \\
\hline & VARIABLE:CONFORT SEGURIDAD PEATONAL & 0.4 \\
\hline \multirow{7}{*}{ 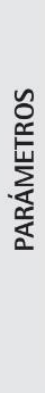 } & - Dotación de mobiliario urbano & 0.05 \\
\hline & - Dimensión y estado de veredas & 0.1 \\
\hline & - Existencia de señalética urbana & 0.05 \\
\hline & - Protección frente al transito vehicular & 0.05 \\
\hline & - Continuidad y claridad de los recorridos & 0.05 \\
\hline & - Conectividad de la red peatonal & 0.05 \\
\hline & - Iluminación & 0.05 \\
\hline \multirow{5}{*}{ 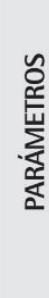 } & VARIABLE: CALIDAD AMBIENTAL & 0.3 \\
\hline & - Calidad y cantidad de arbolado público & 0.1 \\
\hline & - Accesibilidad y proximidad a espacios verdes & 0.1 \\
\hline & - Contaminación Ambiental & 0.1 \\
\hline & TENDENCIA ESPERADA & 1 \\
\hline
\end{tabular}

Fuente: Elaboración propia- GIUR (2020)

Relevancia del indicador

La relevancia del indicador radica fundamentalmente en que permite observar a través de sus distintos parámetros la situación actual del sector analizado en términos de caminabilidad, es decir de las condiciones para caminar de manera cómoda y segura por ese lugar. Detectando, además, áreas potencialmente atractoras de caminantes (aunque esas áreas no posean aún alguna/s características necesarias para transitarlas). Los indicadores y sus criterios de valoración sirven para señalar dónde hay un problema y cuál es la gravedad de la situación con el fin de priorizar las acciones a seguir. "Los hechos son los hechos, pero la realidad es la percepción" (Albert Einstein).

Los resultados de su aplicación permitirán mejorar, en caso de que sea necesario, esas condiciones valorándolas en un contexto físico- espacial- temporal determinado y en el marco de la inclusión social como derecho a la ciudad. "Medir las distancias de las formas urbanas da medida de la capacidad de acceso y de proximidad de la población al espacio que se configura como ámbito urbano" (Llop, 2013). 


\section{Aplicación y Resultados}

En la Tabla $N^{\circ} 4$ se presenta la aplicación del Indicador de Caminabilidad en las dos áreas previamente seleccionadas. Esto es, en el área del microcentro y en un barrio residencial. Las unidades de análisis, son el área y el barrio respectivamente.

Tabla N5- Aplicación del Indicador de Caminabilidad

\begin{tabular}{|c|c|c|c|c|}
\hline \multirow{6}{*}{ : } & INDICADOR DE CAMINABILIDAD & Ponderación & $\begin{array}{c}\text { Área } \\
\text { central } \\
\text { Microcentro }\end{array}$ & $\begin{array}{l}\text { Área de } \\
\text { cercanía } \\
\text { B. del Carmen }\end{array}$ \\
\hline & VARIABLE: MIXTURA DE USOS & 0.3 & 0.29 & 0.14 \\
\hline & \multirow{3}{*}{$\begin{array}{l}\text { - Concentración de actividades } \\
\text { - Densidad de población } \\
\text { - Proximidad de equipamiento }\end{array}$} & 0.1 & 0.1 & 0.01 \\
\hline & & 0.1 & 0.09 & 0.05 \\
\hline & & 0.1 & 0.1 & 0.08 \\
\hline & VARIABLE:CONFORT SEGURIDAD PEATONAL & 0.4 & 0.315 & 0.22 \\
\hline \multirow{7}{*}{ 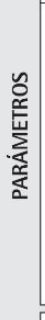 } & \multirow{7}{*}{$\begin{array}{l}\text { - Dotación de mobiliario urbano } \\
\text { - Dimensión y estado de veredas } \\
\text { - Existencia de señalética urbana } \\
\text { - Protección frente al transito vehicular } \\
\text { - Continuidad y claridad de los recorridos } \\
\text { - Conectividad de la red peatonal } \\
\text { - Iluminación }\end{array}$} & 0.05 & 0.04 & 0.02 \\
\hline & & 0.1 & 0.09 & 0.03 \\
\hline & & 0.05 & 0.05 & 0.01 \\
\hline & & 0.05 & 0.025 & 0.05 \\
\hline & & 0.05 & 0.04 & 0.04 \\
\hline & & 0.05 & 0.03 & 0.04 \\
\hline & & 0.05 & 0.04 & 0.03 \\
\hline \multirow{5}{*}{ 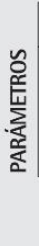 } & VARIABLE: CALIDAD AMBIENTAL & 0.3 & 0.2 & 0.24 \\
\hline & - Calidad y cantidad de arbolado público & 0.1 & 0.07 & 0.08 \\
\hline & - Accesibilidad y proximidad a espacios verdes & 0.1 & 0.1 & 0.08 \\
\hline & - Contaminación Ambiental & 0.1 & 0.03 & 0.08 \\
\hline & TENDENCIA ESPERADA & 1 & 0.80 & 0.60 \\
\hline
\end{tabular}

Fuente: Elaboración propia- GIUR- FAUD-UNSJ

Para poder obtener una "medida" de la aplicación del indicador, se recurre a los rangos de valoración expresados en la Tabla $N^{\circ} 5$.

Tabla Nº- Valoración del Indicador de Caminabilidad

\begin{tabular}{c|ccc|}
\multicolumn{1}{c}{$\begin{array}{c}\text { Valoración } \\
\text { INDICADOR DE }\end{array}$} & BUENO & REGULAR & MALO \\
\cline { 2 - 4 } CAMINABILIDAD & $1-0.7$ & $0.6-0.4$ & $0.3-0.0$ \\
\cline { 4 - 5 } & &
\end{tabular}

Fuente: Elaboración propia- GIUR

La aplicación del Indicador de caminabilidad como un instrumento metodológico, requiere relevamientos in situ de cada variable con sus parámetros, con actualizaciones permanentes para la verificación. En cuanto a los resultados obtenidos, se advierte que en el Área central, sector microcentro, el indicador arroja 0.80 puntos. Ello evidencia de acuerdo a la tabla de valoración, un buen nivel de caminabilidad. Este puntaje se acerca al valor deseado (1) aunque no llega al mismo, 
advirtiéndose que en las tres variables consideradas hay algunos parámetros a mejorar, sobre todo en las variables confort peatonal y contaminación ambiental.

Mientras que, en el sector del barrio del Carmen el indicador obtiene 0.60 puntos, lo que advierte sobre un nivel regular de caminabilidad. Este barrio, tiene similares características a la mayoría de los barrios del Gran San Juan localizados fuera del Área central. Si bien cuenta con equipamiento de salud, educación y comercio diario, no posee grandes atractores para los peatones, de uso social colectivo. Por lo tanto, los parámetros a mejorar están vinculados especialmente a las variables mixtura de usos y confort peatonal.

\section{Algunas aclaraciones respecto a los parámetros considerados}

En la variable Mixtura de Usos, parámetro Densidad de Población: Cabe señalar que en la ciudad de San Juan se verifican densidades de población relativamente bajas, en comparación a otras ciudades de similares características y escala. Si bien la densidad es un factor que varía notablemente de una ciudad a otra, se toma como una graduación relativa dentro de cada ciudad. En el caso de San Juan, como ciudad localizada en zona sísmica y con escasez de agua, la tendencia debería ser hacia una densidad Media, aunque esto no se verifica en gran parte de la ciudad, siendo los valores promedio por debajo de 50 habitantes/Ha, sobre todo en las zonas residenciales fuera del casco urbano (GIUR- FAUD-UNSJ, 2014-2015).

En cuanto a la variable "Confort peatonal", y su parámetro de ancho y estado de las veredas, cabe aclarar: En el área central de la ciudad, especialmente en el casco urbano, la ciudad cuenta con veredas muy espaciosas, fruto de la remodelación postterremoto de 1944. Las amplias veredas responden a la necesidad de "escape" frente a un evento sísmico, tal como fueron concebidas en los Planes de reconstrucción. "Los sucesivos Planes Oficiales de reconstrucción de la ciudad, plantearon nuevos perfiles de calles que tienen en cuenta aceras amplias con cunetas de riego y arbolado" (Riotman D., 1996).

De igual modo, los parámetros destinados a valorar el estado y ancho de las veredas, la señalización, claridad y conectividad de los recorridos, implican la consideración de todos los transeúntes, garantizando la accesibilidad como la posibilidad que tienen las personas con o sin problemas de movilidad para recorrer el espacio urbano. Aquí se tiene en cuenta además, la disponibilidad de rampas para discapacitados. La presencia de estos elementos es mayor en el área central que en los barrios adyacentes, donde es incipiente su construcción en las esquinas de las calles 
principales, y en otras zonas residenciales como el barrio del Carmen, son elementos inexistentes.

En la variable Calidad Ambiental, el arbolado público en cuanto a su cantidad y calidad, es fundamental en esta ciudad. La arboleda urbana plantada regularmente a ambos lados de las calles, conforman una verdadera pantalla que da uniformidad al conjunto, a modo de fachada continua. Las acequias, canales regadores de los espacios públicos y del arbolado urbano, conforman una red similar a la empleada para el riego agrícola del área rural, con un trazado coincidente con el de las calles (Nacif, N. et al, 2011).

\section{Relevamiento fotográfico del Microcentro Comercial, Área peatonal.}

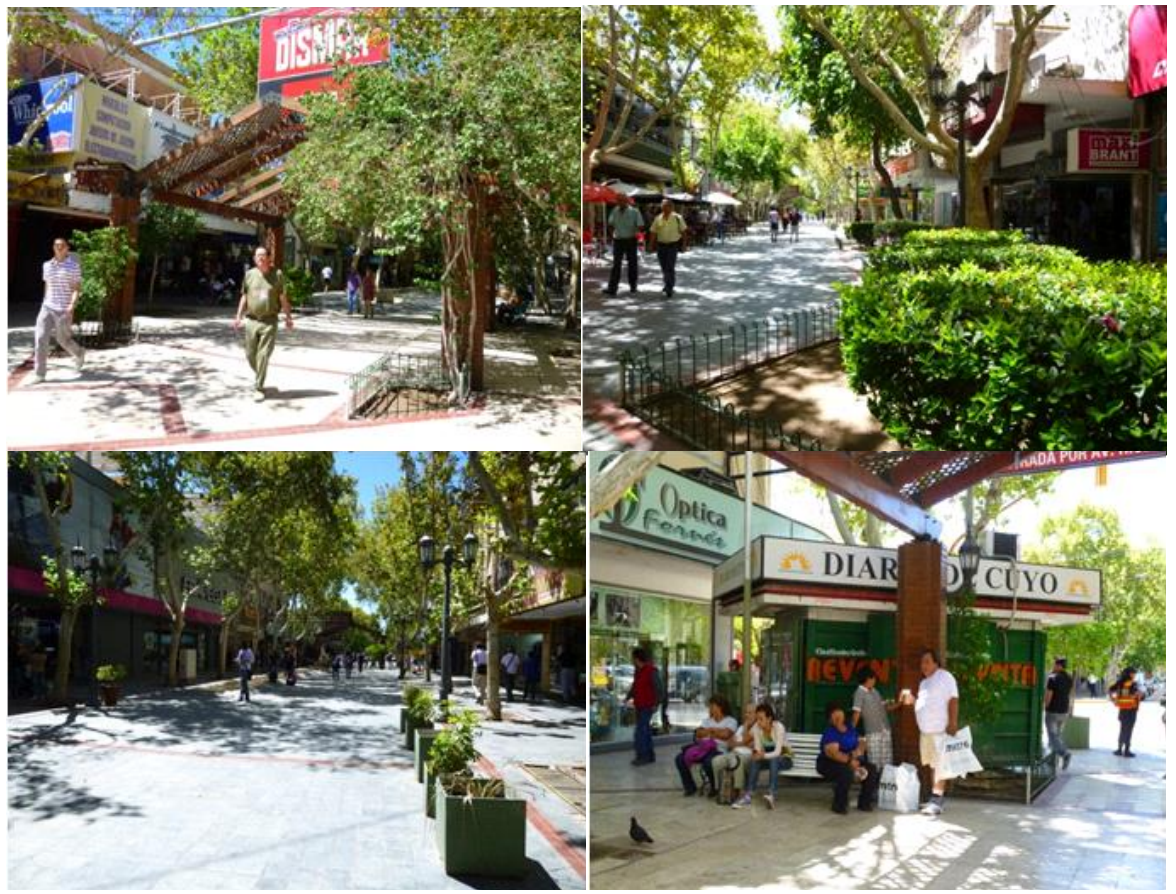

Fuente: Archivo propio del Gabinete de Investigaciones Urbanas- GIUR 


\section{Relevamiento fotográfico del Barrio del Carmen}

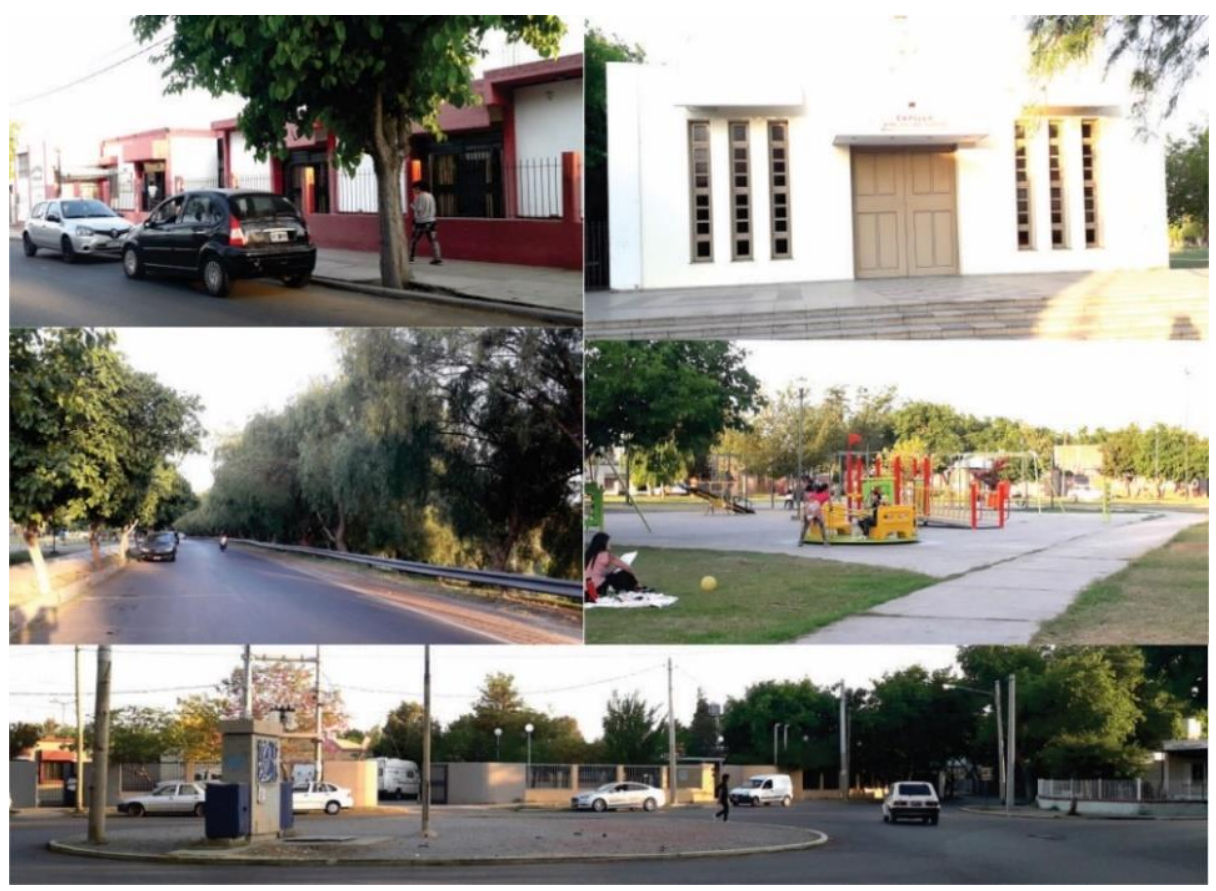

Fuente: Archivo propio del Gabinete de Investigaciones Urbanas- GIUR

\section{Consideraciones finales}

A lo largo del presente trabajo se ha intentado demostrar la importancia de la caminabilidad desde la construcción de un indicador que coadyuve a su visibilización como un derecho y un aporte al desarrollo sustentable.

El desarrollo sustentable e inclusivo de las ciudades está intrínsecamente vinculado al derecho a una ciudad caminable. En términos de derechos "Las ciudades deben garantizar a todas las personas el derecho de movilidad y circulación en la ciudad" (Carta mundial del Derecho a la ciudad, 2004).

La dependencia del transporte automotor en el último siglo, ha tenido causas y consecuencias diversas, aunque se advierte en la actualidad un incipiente cambio de mentalidad (o tal vez de paradigma), a través de conductas favorables a otros estilos de vida, que incluyen el hábito de caminar. Los avances en el conocimiento y el mayor acceso a la información sobre las problemáticas ambientales, va llevando a la población a la adopción de actitudes y prácticas más saludables, lo que a su vez tiene derivaciones e incidencia en la integración y cohesión social.

En las zonas más caminables desaparecen las incomodidades derivadas de un gran volumen y velocidad de tránsito, el riesgo en la seguridad del peatón y la contaminación atmosférica, mejorando consecuentemente el confort térmico y el paisaje urbano. 
Actualmente se acepta que el cuidado de la vida urbana y de las personas en el espacio público debe jugar un rol central a la hora de planificar ciudades y áreas urbanas (Gehl, J. 2014). Cualquier modelo de ciudad deseada debe contemplar acciones para obtener óptimas condiciones ambientales, este interés se incrementa en ciudades con las características de la ciudad de San Juan, cuyo medio natural le impone condicionantes a los que la población debe adaptarse.

La contribución de este estudio, se centra en proporcionar elementos útiles para ser considerados en Planes Urbano Ambientales que atiendan a la caminabilidad como un valor y como un derecho urbano de la población. El indicador aportado pretende ser una herramienta como dispositivo de prevención y asimismo, facilitar en etapas posteriores un mecanismo de control para ser aplicado en diversos sectores de la ciudad.

En este proyecto se trabajó con variables y parámetros generales para la conformación del indicador, aunque se debe aclarar que dentro de ellos, o derivados de los mismos, se pueden generar sub- indicadores, (por ejemplo para distintos momentos del día), con los que se podría atender a otros aspectos de la problemática dentro de una mirada integral de la ciudad. En ese sentido, este indicador de caminabilidad propuesto, integra un sistema de indicadores cuyo funcionamiento debe asumir una dinámica de permanente revisión, que permita mejorar, corregir y/o renovar su ejercicio. Es decir, debe tenerse en cuenta como un instrumento orientador y al servicio de la gestión pública, en un proceso dinámico, constante, continuo y complejo.

Por último, se afirma que el actual contexto de pandemia resignifica todos los conceptos relativos a la cuestión urbana, interpelando al diseño y al planeamiento urbano tradicionales e imponiendo una visión sobre las ciudades con otros objetivos orientados a mejorar significativamente la calidad de vida de las personas. El espacio público caminable debe configurarse y entenderse entonces, como un ámbito con valor social, ambiental, económico, paisajístico y saludable que posibilite la integración social y urbana de toda la ciudad. 


\section{Referencias Bibliográficas}

AGENCIA DE ECOLOGÍA URBANA DE BARCELONA (2011). Sistema de Indicadores y Condicionantes para ciudades Grandes y Medianas. Documento PDF. Ministerio de Medio Ambiente y Medio Rural y Marino. Barcelona, España.

ÁLVAREZ DE CELIS, F.; ÁLVAREZ INSÚA J.; EGUÍA S.; PERSICO M. E.; BELACIN S.; TRÍPOLI F. (2014). Índice sintético de caminabilidad. Metodología. PDF. Ministerio de Desarrollo Urbano. Gobierno de la Ciudad de Buenos Aires.

CARRIÓN, F. DAMMERT-GUARDIA, M. (2019) Derecho a la ciudad: una evocación de las transformaciones urbanas en América Latina / 1a ed. - Lima: CLACSO, Flacso http://biblioteca.clacso.edu.ar/clacso/gt/20200519104921/Derecho-a-la-ciudad.pdf. Ecuador, IFEA, ISBN 978-612-4358-05-0.

COALICIÓN INTERNACIONAL PARA EL DERECHO A LA CIUDAD (2004) "Carta Mundial por el Derecho a la Ciudad". Disponible en: file://C:/Users/PERSONAL/Downloads/HIC-Document-5785-20181217-2134.pdf

CORRALIZA RODRÍGUEZ, J.A.; BERENGUER, S.; MARTíN, R. (2006). "Medio ambiente, bienestar humano y responsabilidad ecológica". Edit. Resma. Madrid, España. ISBN 978-84-935532-0-3

FALÚ, A. M. (2014). "El derecho de las mujeres a la ciudad. Espacios públicos sin discriminaciones y violencias". Revista Vivienda y Ciudad - ISSN 2422-670X -Vol. 1.

FOLCH R. Y BRU J. (2017) "Ambiente, territorio y paisaje. Valores y valoraciones". Editorial Barcino. Barcelona/Madrid. ISBN: 978-84-7226-819-7

GARCÍA DELGADO D. (2020). Ciudad y pandemia: las metrópolis en cuestión. https://www.flacso.org.ar/noticias/ciudad-y-pandemia-las-metropolis-en-cuestion/

GEHL, J. (2014) Cities for people. ISBN 978-987-9393-80-2. ONU-Hábitat. Ed.Infinito GIUR-FAUD-UNSJ (2014-2015). Estudio de Indicadores de Sustentabilidad Urbana. Aplicación en áreas críticas de la ciudad de San Juan. Proyecto de Investigación. Dirección: Nacif N.

GUTIÉRREZ, D. (2009). La Construcción de Indicadores como Problema Epistemológico Cinta Moebio 34:16-36 www.moebio.uchile.cl/34/gutierrez.html 
GUTIÉRREZ LÓPEZ, CABALLERO PÉREZ, ESCAMILLA TRIANA, (2019). Índice de caminabilidad para la ciudad de Bogotá. Revista de Arquitectura (Bogotá), 21(I), 8-20. doi: http://dx.doi.org/10.14718/RevArq.2019.21.1.1884

JACOBS, JANE (1961). "The Death and Life of Great American Cities". Citado en "Indice sintético de caminabilidad" 2011, Gobierno de CABA. Buenos Aires

LEVA, GERMÁN (2005). Indicadores de calidad de vida urbana. PDF. UNQuilmesHábitat/Metrópolis

LITMAN, TODD (2011) "Developing Indicators For Comprehensive And Sustainable Transport Planning". https://www.vtpi.org/sus_tran_ind.pdf

LLOP, JOSEP Ma. (2013). Medir la sostenibilidad urbana a escalas intermedias: Compacidad-proximidad y habitabilidad-accesibilidad. Revista Catalana de Acceso Abierto: http://www.raco.cat/index.php/Sostenible/article/view/261968

NACIF N., ESPINOSA M, MARTINET M (2012). Indicadores para evaluación de la sustentabilidad en la ciudad de San Juan, Argentina. Revista ANDINAS, Revista de Estudios Culturales en torno a la Arquitectura, el Urbanismo y el Diseño. Volumen: 012012, Página: 56 a 63 - Publicación con Referato - Código ISSN: 2250-4931

NACIF N., ESPINOSA M, MARTINET M (2011). Una ciudad oasis de zona sísmica. Revista Iberoamericana de Urbanismo (RIURB). 1 Setembre 2011, núm. 6, p. 7380. URIhttp://hdl.handle.net/2099/12525. ISSN2013-6242

NACIF N. (2017) "Diseño de indicadores urbanos de sustentabilidad. El caso del Gran San Juan en Argentina". Revista Urbano № 34. Pág.6 - 15. ISSN 0717 - 3997 / 0718 3607. Universidad Bio-Bio, Chile.

NUÑEZ, ANA (2016): Del Derecho a la Ciudad, al Derecho al Espacio Diferencial. Revista de Direito da Cidade, vol.06, nํ01. ISSN 2317-7721 p.90-110. Brasil

ONU-HÁBITAT (2016) "Nueva Agenda Urbana". Disponible en: https://onuhabitat.org.mx/index.php/la-nueva-agenda-urbana-en-espanol.

ONU-HÁBITAT (2021) "Los usos mixtos del suelo y sus beneficios". https://onuhabitat.org.mx/index.php/los-usos-mixtos-del-suelo-y-sus-beneficios 
ROITMAN, DORA y otros. San Juan, La ciudad y el Oasis. Editorial EFU - San Juan, Argentina. 1996.

RUEDA PALENZUELA, S. (1999). Modelos e Indicadores para ciudades más sostenibles. PDF. Taller sobre Indicadores de Huella y Calidad Ambiental Urbana. Generalitat de Catalunya.

SÁNCHEZ DE MADARIAGA, INÉS (2008). Urbanismo con perspectiva de género. Ed. Instituto Andaluz de la mujer. ISBN: 847921-101-6. https://www.juntadeandalucia.es/institutodelamujer/ugen/sites/default/files/documentos/ 98.pdf

UN-HÁBITAT, 2015- Transformar nuestro mundo: la Agenda 2030 para el Desarrollo Sostenible (documento A/70/L.1), 18 /09/ 2015.

SENTAGNE M.E., SOLERA E., ROSES M. Y LACIAR M. (2010). La imagen moderna de la ciudad de San Juan. VI Jornada de Sociología de la UNLP. PDF. La Plata, Argentina

ZICCARDI, A. y otros (2017): Ciudades sostenibles y derechos humanos. p1. Universidad Nacional Autónoma de México. Coordinación de Humanidades Programa Universitario de Estudios sobre la Ciudad. México.

https://www.cndh.org.mx/sites/all/doc/Informes/Especiales/Ciudades-Sostenibles-DH. pdf 